\title{
Dynamic Optimization Model of Signal Timings of Exit-lanes for Left-turn Intersections
}

\author{
Kun Yang, Yinzhen Li, Hao Li and Jie Yin \\ Lanzhou Jiaotong University, School of Transportation, Lanzhou Gansu China 730000
}

\begin{abstract}
To make use of the space and time resources at the conventional intersections using exit-lanes for left-turn (EFL) effectively, a dynamic signal-timing model for such special intersections was proposed. Firstly, the operator of the optimal period of the main signal lamp was determined by means of calculating the mean value. The concept of coordination timing of the main signal and pre-signal lights was discussed. Then the model was established with the opening and closing time of the pre-signal as the constraints, and average traffic delay as the objective function. Finally, the validity of the model was tested by taking the crossroad of Jingshi Road and Shungeng Road In Jinan City as an example. Its model was solved by the method of Enumeration with $C$ programming. The results show that the average delay of vehicles passing through the intersection is reduced by $13.74 \%$ by dynamically adjusting the signal timing of the intersection. The timing model can make efficient utilization of the space-time resources at the intersection, thus improving the traffic capacity of the intersection.
\end{abstract}

Keywords - traffic management; traffic organization; exit-lane for left-turn; dynamic timing model

\section{INTRODUCTION}

The traffic capacity of the intersection of the urban road network restricts the capacity of the entire road network. To improve the traffic capacity of the intersection is one of the effective ways to ease the traffic congestion in the city. In order to improve public transport services, the British Transport Authority first proposed the concept of pre-signal control in a report on the promotion of public transport development in 1991.Wu and Hounsell et al[1-2] proposed a method of setting up pre-signal at the upstream of intersections to reduce the interaction between bus and private cars at intersections. Countries such as the United States, Switzerland and Germany use this method for bus lanes to reduce delays of buses at intersections. Tian Yunqiang et al[3] combined the exit lanes at the intersection of Jingshi Road and Luneng Road in Jinan, then analyzed and studied the parameters and corresponding supporting measures such as the setting constraints of the intersection, the number of lanes in exit-lanes for left-turning, the length of the lane, and the minimum time for opening and closing of pre-signal. In the article, he mentioned three types of adjustments of main signal and lane function: (i)We keep signal cycle length unchanged, to shorten the time of the left-turn green light and increase the ratio of straight green signal to main signal cycle length;(ii)To shorten the signal cycle, reduce the time of the left-turn green light and optimize the ratio of straight green signal to main signal cycle length;(iii)To adjust the lane function, change the allocation of space rights, and re-design the signal timing plan. However, he did not conduct in-depth analysis of the three methods. Ma Wanjing, Zhao Jing, et al[4-5] made maximum reserve capacity as objective function, and Consider the constraints such as traffic flow, division of lane function, main signal control, pre-signal control, length of the integrated functional area, and saturation to establish a mixed integer nonlinear programming model. Then they use the branch and bound method to solve it. The models established in the above studies are all fixed signal timing, but the capacity of the intersection is not fully released for the changing traffic flow. So, the establishment of a dynamic optimization model can make better use of intersection space and time resources and improve the traffic capacity of intersection.

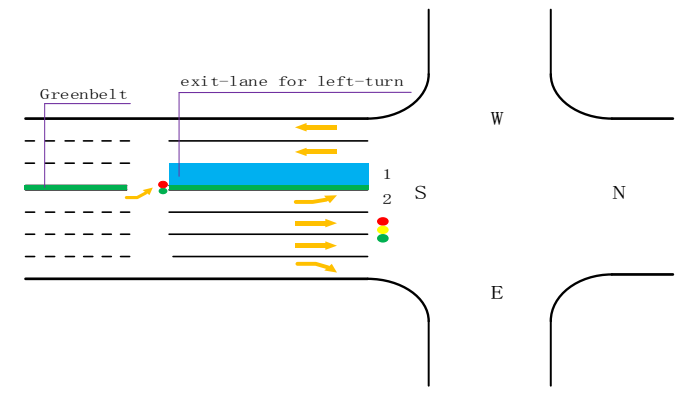

FIGURE I. BASIC CONCEPTS OF EXIT-LANES FOR LEFT-TURN INTERSECTION

\section{BASIC CONCEPT}

In order to illustrate the basic concept of exit-lanes for left-turn intersections, the intersection of Jingshi Road and
Shungeng Road in Jinan City was taken as an example. Table 1 shows the specific canalization methods for imports in all directions. The green part is the central reserve, and the blue part exit-lane for left-turn at figure 1 . At a certain distance 
from the intersection, the greenbelt is opened with a pre-signal light set to control whether the left-turn vehicles are allowed to enter the exit line. When the green light of the pre-signal light is on, the left-turning vehicle at southern entrance can enter the exit line to prepare for left-turning. While the red light is on, the exit line acts itself role to allow else vehicles leaving away from the intersection. The reconstruction of the intersection design method has a small workload, and has less adverse impact on other traffic flows. At the same time, the method makes the left-turning vehicles get extra road right and reduces delay of vehicles at the intersection.

TABLE I. CHANNELIZATION TABLES AT THE CROSSROAD OF JINGSHI ROAD AND SHUNGENG ROAD IN JINAN CITY

\begin{tabular}{|c|c|c|c|c|c|}
\hline \multirow{2}{*}{ Derections } & \multicolumn{4}{|c|}{ Canalization of Imported Lanes } & exit lanes \\
\cline { 2 - 5 } & left-turning lanes & straight lanes & right-turning lanes & bus lanes & \\
\hline East & 1 & 4 & 1 & 1 & 4 \\
\hline South & 1 & 2 & 1 & 0 & 3 \\
\hline West & 1 & 4 & 1 & 0 & 4 \\
\hline North & 1 & 2 & 1 & 0 \\
\hline
\end{tabular}

\section{DYNAMIC TIMING MODEL}

\section{A. Parameters}

$\mathrm{L}$ the length of exit lane for left-turning.

1 the sum of the time lost at one signal cycle.

I time loss of starting vehicle.

A yellow time.

$y_{i j} \quad$ saturation of the $\mathrm{j}$-th lane of the i-th phase.

$Q_{N} \quad$ traffic volume of Nth entrance.

$\mathrm{E}(\mathrm{Q})$ mean value of traffic volume for all entrances.

$\beta \quad$ boundary value.

$\mathrm{P}$ the amount of traffic signal phase

$V_{e} \quad$ maximum equivalent traffic flow at all single lanes.

M equivalent traffic flow at peak hour for right-turning and straight lanes.

$\mathrm{N}$ traffic flow at left-turning lanes.

$G_{p g} \quad$ duration of pre-signal green light.

$g_{m} \quad$ duration of main-signal red light.

$t_{1} \quad$ opening time of pre-signal in advance

$t_{2} \quad$ closing time of pre-signal in advance

$G_{p r} \quad$ duration of pre-signal red light.

$C_{p} \quad$ the cycle length of pre-signal

$V_{0} \quad$ average speed vehicles passing through the intersection.

\section{B. Decision Variables}

$C_{d} \quad$ the cycle length of main signal. $q_{i} \quad$ saturation flow rate.

\section{Objective Function}

In order to find the non-inferior solution and simplify the process of solving, the average delay of each entrance was chosen from all evaluation index of signal timing with reference to Webster's delay formula [9]:

$$
F_{\mathrm{i}}=\frac{C_{d} \times\left(1-\lambda_{i}\right)^{2}}{2 \times\left(1-\lambda_{i} \times y_{i}\right)}+\frac{y_{i}{ }^{2}}{2 q_{i} \times\left(1-q_{i}\right)}-0.65 \times\left(\frac{C_{d}}{q_{i}}\right)^{\frac{1}{3}} \times y^{2+5 \lambda_{i}}(1)
$$

The objective function of total average delay of all vehicle passing through the intersection can be represented by

$$
\operatorname{Min} D=\sum_{i} F_{i}
$$

\section{Parameters of Main Signal}

For an independent intersection with stable traffic flow, if the traffic flows at all entrances are equal and the arrival time of every vehicle is random, according to an experimental study by the British TRRL [6], the formula of cycle length for the minimum average delay of vehicles at intersections is

$$
\begin{gathered}
C_{\mathrm{d}}=\frac{1.5 L+5}{1-Y} \\
L=\sum(l+I-A) \\
Y=\sum_{i} \max \left(y_{i j}\right) \leq 0.9
\end{gathered}
$$

However, in the actual traffic survey, it is impossible to have absolutely equal traffic flows at each entrance. We can relax harsh conditions. This formula can be used when there is little difference between all traffic flows at each entrance. we first calculate the mean value of all traffic flows. The absolute value of traffic flow at an entrance minus the mean value qualifies little difference. The mathematical expressions are 


$$
\begin{gathered}
E(Q)=\frac{\sum_{1}^{4} Q_{N}}{4} \\
\left|Q_{N}-E(Q)\right| \leq \beta
\end{gathered}
$$

When the value of $\beta$ is determined, if equation (7) holds for any $\mathrm{N}$, we can use Equation (3) to calculate the signal cycle length. If not, we can use Equation (8).

$$
\begin{gathered}
C_{\mathrm{d}}=\frac{13330 P}{1333-V_{e}} \\
V_{\mathrm{e}}=\frac{V+0.5 M+0.6 N}{B}
\end{gathered}
$$

The cycle length of the main signal should not be too short, otherwise the traffic at the intersection will be chaotic. Excessive cycle length results in increased delay at the intersection. The value range of signal cycle length is

$$
C_{\min } \leq C_{d} \leq C_{\max }
$$

$C_{\text {min }}$ represents the minimum value of signal cycle length. $C_{\max }$ stands for the maximum value of signal cycle length. The green time of all phases are assigned by the proportion of the actual traffic flow [8].

\section{E. Parameters of Pre-signal}

The pre-signal is to organize the traffic in advance and needs to coordinate with the main signal. The cyclic relationship between the two is shown in Figure 2. Therefore, the pre-signal cycle length $C_{p}$ should be equal to the cycle length of the main signal $C_{d}$, ie: $C_{P}=C_{d}$. The pre-signal cycle length consists of the green light length and the red light length. The length of green light is

$$
G_{p g}=g_{m}+t_{1}-t_{2}
$$

The length of red light is

$$
G_{P R}=C_{P}-G_{P G}
$$

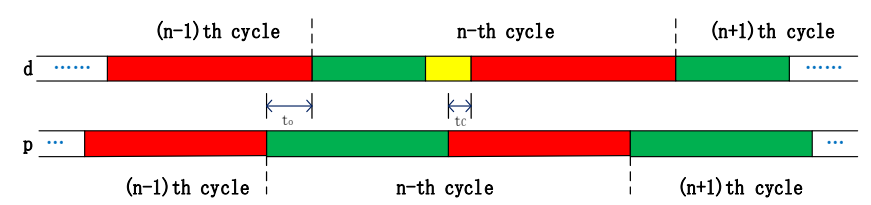

FIGURE II. CYCLE DIAGRAM OF PRE-SIGNAL LIGHT AND LEFT-TURNING PHASE OF MAIN LIGHT AT AN ENTRANCE (d represents the left-turning phase of the main signal, $p$ represents the pre-signal light)

Before the green light of the left-turn phase at southern entrance is turned on, left-turning vehicles in lanes 1 and 2 in Figure 1 need to be queued in advance to prepare for left turning. Vehicles in lane 2 are only controlled by the main signal, so they can be queued after they have successfully reached the parking line. However, vehicles in lane 1 are also controlled by pre-signal. In order for vehicles entering Lane 1 to wait in line after the parking line, green light of the pre-signal should turn on before the left-turning green light of main signal does. Since Lane 1 also serves as the exit lane, there will be reversed traffic in the lane after the green lights at other phases turn on. Therefore, the green light of the pre-signal should be turned off early to ensure that the last vehicle entering the 1st lane can safely pass through the intersection at the end of the left-turning green light of main signal. Phase cycle relationship of pre-signal signal and left-turning light of main signal shown in Figure 2. The figure shows the time difference between the opening and closing of the main and pre-signal lights in the n-th cycle. $t_{1}$ is the time for the pre-signal to be turned on in advance, and $t_{2}$ to be turned off in advance. The conditions that should be satisfied by $t_{1}$ and $t_{2}$ respectively are

$$
\begin{gathered}
\frac{L}{V_{0}}+t_{0} \leq t_{1} \leq C_{d}-g_{m} \\
t_{0}+\frac{L}{V_{0}}+t_{n} \leq t_{2} \leq g_{m}
\end{gathered}
$$

\section{SUPPORTING MEASURES}

\section{A. Detectors Layout}

In this section, we first choose the four directions of the intersection, where have straight lines and left lanes. According to traffic rules, the right line will not be considered. The upstream and downstream (stop line) of each lane are equipped with signal detectors to form test area. The upstream detectors are used to detect the total amount of vehicles arriving and the downstream detectors to detect the total amount of vehicles leaving. Detectors monitor dynamically traffic flow of each lane and other traffic information to provide the necessary data for control system.

\section{B. System Operating Principle}

After the system receives the data, the initial delay $D_{0}$ of $\mathrm{n}$ cycles of the first statistical cycle can be counted and then calculate delay $D_{1}$ after optimizing in the next n cycles.

$$
\left(D_{1}-D_{0}\right) / D_{0} \geq \gamma>0
$$




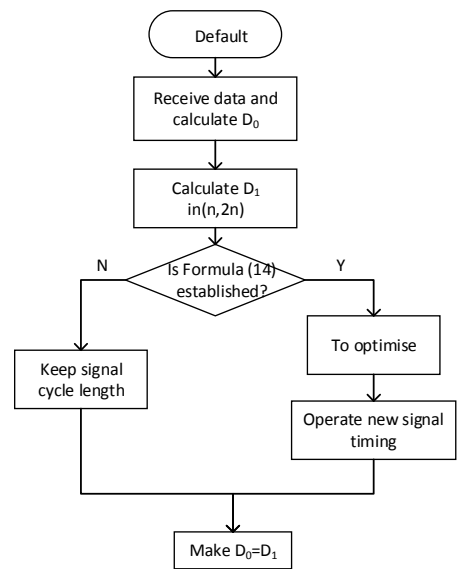

FIGURE III. DYNAMIC TIMING PRINCIPLE OF SYSTEM

If equation (15) holds, we can use the model of second chapter to recalculate the signal timing of the intersection. The optimizing signal cycle is transmitted to the signal machine at the $(2 n+1)$ th cycle and begins at the $(2 n+2)$ th cycle. If equation (15) does not hold. The signal cycle keeps unchanged. At last we make $D_{0}=D_{1}$. The system operate above process one by one. The main signal and the pre-signal are Regularly optimized with the traffic flow. It will cause the vehicle delay to decrease. The principle of system operating shown in Figure 3.

\section{TIME COMPLEXITY ANALYSIS AND CALCULATION EXAMPLES}

From the figure 3 analyzing the procedure of optimizing dynamically the signal cycle, it is independent of the traffic flow. The total steps of executing the algorithm is a constant, so the time complexity is $\mathrm{O}(1)$.

TABLE II. COLLECTED TRAFFIC FLOW DATA AT THE INTERSECTION OF JINGSHI ROAD AND SHUNGEN ROAD IN JINAN CITY

\begin{tabular}{l|llll} 
Direction/flow & $\begin{array}{l}\text { East } \\
\text { entrance }\end{array}$ & $\begin{array}{l}\text { South } \\
\text { entrance }\end{array}$ & $\begin{array}{l}\text { West } \\
\text { entrance }\end{array}$ & $\begin{array}{l}\text { north } \\
\text { entrance }\end{array}$ \\
\hline $\begin{array}{l}\text { Direct number of } \\
\text { vehicles }\end{array}$ & 2933 & 551 & 2546 & 673 \\
$\begin{array}{l}\text { Turning left } \\
\text { Turning right }\end{array}$ & 345 & 488 & 885 & 318 \\
$\begin{array}{l}\text { The total traffic } \\
\text { volum }\end{array}$ & 3502 & 1384 & 346 & 211 \\
\hline
\end{tabular}

volume

First, supposed that the condition of formula (13) holds, we need to optimize signal cycle in $(2 n+2,3 n+2)$. the data of Table 2 was collected from the crossroad of Shungen Road and Jinshi Road in the city of Jinan. Its signal cycle length is 200s. The monitoring time was an hour, so $n=60 * 60 / 200=18$.
According to the investigation and analysis, the average speed of the intersections is $25 \mathrm{~km} / \mathrm{h}$. The main signal cycle length is an integer in the range of 25s and 300s. The longer the signal cycle length is, the longer the delay is. After optimizing the signal cycle, its value is often down. Therefore, the optimization interval is in $\left(C_{\min }, C_{P}\right)$.Therefore, the whole solution process is an integer-nonlinear programming with finite intervals, which can be solved by programming enumeration.

TABLE III. OPTIMIZING RESULTS OF MAIN SIGNAL CYCLE

\begin{tabular}{|l|l|l|l|l|l|}
\hline Timing & $\begin{array}{l}\text { straight } \\
\text { (east } \\
\text { and } \\
\text { west) }\end{array}$ & $\begin{array}{l}\text { left } \\
\text { (east } \\
\text { and } \\
\text { west) }\end{array}$ & $\begin{array}{l}\text { straight } \\
\text { (north } \\
\text { and } \\
\text { south) }\end{array}$ & $\begin{array}{l}\text { left } \\
\text { (north } \\
\text { and } \\
\text { south) }\end{array}$ & $\begin{array}{l}\text { signal } \\
\text { cycle } \\
\text { length }\end{array}$ \\
\hline $\begin{array}{l}\text { Original } \\
\text { timing ( s ) }\end{array}$ & 89 & 30 & 37 & 32 & 200 \\
\hline $\begin{array}{l}\text { Optimizing } \\
\text { timing ( s ) }\end{array}$ & 52 & 39 & 30 & 28 & 160 \\
\hline
\end{tabular}

After solving the example of model, the opening time and closing time of pre-signal at the south entrance are $5 \mathrm{~s}$ and $6 \mathrm{~s}$ respectively and the signal cycle length is 131s. As shown in table 3 , the total length of the signal cycle is reduced by 40 seconds. The green time of the four phases are reduced by $37 \mathrm{~s}$, $9 \mathrm{~s}, 7 \mathrm{~s}$ and $4 \mathrm{~s}$ respectively. The average delays of entrances were reduced by $14.87 \%, 36.77 \%, 20.55 \%$, and $23.31 \%$ respectively and shown in figure 4.

\section{CONCLUSIONS}

In this paper, the dynamic optimization model of exit lane for left-turning intersection with objective function of total average delays is used to optimize signal cycle length. According to the calculating results, the delay of the whole intersection was reduced by $13.74 \%$. Therefore, the time and space resources of such special intersections can be effectively utilized to improve the capacity of intersections. However, there are still some problems to be solved: (1) In this paper, only four-phase intersections have been studied. In the future, other forms of intersections and phase timing can be further studied (eg, Y-shaped intersection, overlapping phases; (2) There are many indicators for evaluating intersections (eg, queue length, capacity, delay, density, etc.), and the Model with a single objective function is insufficient. In future studies, the evaluation of intersections should be considered comprehensively. 


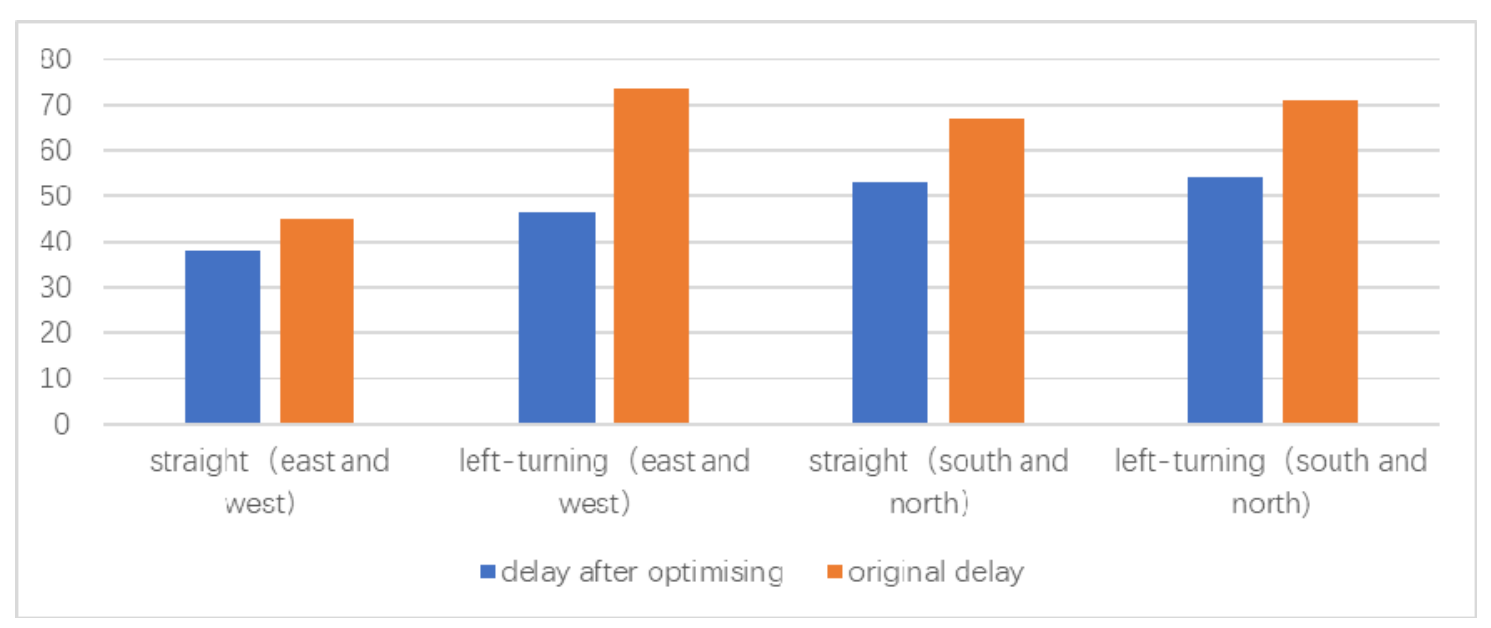

FIGURE IV. COMPARISON OF ORIGINAL DELAY AND ONE AFTER OPTIMIZING

\section{ACKNOWLEDGMENT}

Firstly, I thank my tutor Yinzhen Li very much. He is knowledgeable and kind. Then Li Hao and Yin Jie did much work for the paper. I express my heartfelt thanks to you. Lastly, Thanks to the reviewers organizers of the meeting.

\section{REFERENCES}

[1] Wu J, Hounsell N. Bus Priority Using pre-signals[J]. Transportation Research Part A Policy \& Practice, 1998, 32(8):563-583.

[2] Xuan Y, Gayah V, Daganzo C, et al. Multimodal Traffic at Isolated Signalized Intersections: New Management Strategies to Increase Capacity[J]. 2009(1039183).

[3] Tian Yunqiang, Shang Zhenhua. Design of Reversible Exit Lanes at Urban At-grade Intersections [J]. urban communications, 2014(1):74-80.

[4] Zhao Jing, Ma Wanjing, Han Yin. Method of Intersection Traffic Control Using Exit Road to Turn Left. CN103871256A[P]. 2014.

[5] Zhao Jing, Ma Wanjing, Han Yin. Integrated Optimization Model of Layouts and Signal Timing of Exit-Lanes for Left-Turning Intersection[J]. China Journal of Highway and Transport, 2017, 30(2):120-127.

[6] Xu Jiqian, Chen Xuewu. General Introduction to Traffic Engineering [M]. People's Communication Publishing Co., Ltd., 2008:197-198.

[7] Gallivan S, Heydecker B. Optimising the control performance of traffic signals at a single junction[J]. Transportation Research Part B, 1988, 22(5):357-370.

[8] Cai Jinde. On the Signal Timing optimization of Urban road Optimization[D]. Beijing Jiaotong University, 2012:35-36.

[9] Hummer J E. Unconventional Left-Turn Alternatives for Urban and Suburban Arterials--Part One[J].ITE Journal, 1998,68(9):26--29.

[10] Hughes W, Jagannathan R, Sengupta D, et al. Alternative Intersections / Interchanges : Informational Report (AIIR)[J]. Costs, 2010. 